\title{
Manifestations crâniofaciales et dentaires de la pseudohypoparathyroïdie : une observation exceptionnelle
}

\author{
Craniofacial and dental manifestations in a case of pseudohypoparathyroidism: \\ an exemplary observation
}

FRANÇOIS GEORGES WÉMEAU*, VIRGINIE VLAEMINCK-GUILLEM*

\begin{abstract}
RÉSUMÉ
La pseudohypoparathyrö̈die est une affection héréditaire rare, caractérisée par des résistances hormonales multiples. L'examen clinique et radiologique d'une patiente de 26 ans, atteinte d'une pseudohypoparathyroïdie, montre un sujet brachycéphale, euryprosope (du grec : eurus, large et prosopsis, visage) et transfrontal, avec un étage moyen de la face diminué, des arcades dentaires larges, des diastèmes, des dents hypoplasiques avec des racines courtes et des pulpes larges ainsi qu'un prognathisme (classe Ill squelettique) majoré par une propulsion spontanée due à l'absence de calage postérieur bilatéral. La mandibule est courte, arquée, ancrée postérieurement, avec un champ mandibulaire développé, associée à une brachymaxillie ; les corticales des os du crâne sont denses, la pneumatisation des sinus importante.

Une image radioclaire, peu dense et large, située entre les deux incisives centrales maxillaires fait évoquer un kyste de la papille palatine. (Med Buccale Chir Buccale 2006; 12: 83-88).
\end{abstract}

médecine buccale chirurgie buccale

VOL. $12, \mathrm{~N}^{\circ} 2$ 2006

page 83

mots clés : PTH, PTHrP, dents, pseudohypoparathyroïdie, radiographie

\section{SUMMARY}

Pseudohypoparathyroidism is a rare hereditary disease characterized by a multiple hormonal resistance. Clinical and radiographic investigations concerning a 26 years old woman have shown a brachycephal, euryprosope and transfrontal subject with a middle level of the face reduced, broad dental arches, diastemas, hypoplasias, short roots with denticles and a prognatism lincked with the skeletal classe III and the propulsion of the jaw because of the loss of posterior wedging. The jaw is short, curved, in posterior position with an augmentation of the mandibular field and a brachymaxilly; the corticals of the bone of the skull are dense, the pneumatisation is important with broad sinus.

A clear radiographic image, broad and not very dense can be seen between the two maxillary central incisors which can refer to a cyst of the palatin papilla. (Med Buccale Chir Buccale 2006; 12: 83-88).

key words : PTH, PTHrP, teeth, pseudohypoparathyroidism, radiograph

\footnotetext{
* Service de Médecine Interne et d'Endocrinologie, Clinique Marc Linquette, USN A, CHU de Lille, 59037 France

Demande de tirés à part :

François Georges Wémeau Clinique Marc Linquette 6 rue du Professeur Laguesse 59037 Lille e-mail : wemeau@hotmail.com 
médecine

buccale

chirurgie

buccale

VOL. $12, \mathrm{~N}^{\circ} 2$ 2006

page 84
La pseudohypoparathyroïdie (PHP) est une affection héréditaire rare dont la fréquence semble être cependant sous-estimée. Albright et coll. en ont fait la première description en 1942 évoquant déjà la notion de résistance hormonale ${ }^{[1]}$. Elle se traduit par la résistance des tissus cibles à l'action de la parathormone (PTH), hormone hypercalcémiante produite par les glandes parathyroïdes. On distingue différentes formes de PHP répondant à une physiopathologie différente, basée sur la résistance rénale à la PTH : le type I (avec les sous-types la, lb et Ic) et le type II [26]. A côté de ces deux types principaux, il existe la pseudopseudohypoparathyroïdie, souvent assimilée à la PHP de type la. La forme la plus courante, la PHP de type la ou ostéodystrophie héréditaire d'Albright $(\mathrm{OHA})$, est caractérisée par différents signes cliniques : une petite taille, une obésité, un retard mental fréquent d'intensité faible ou modérée, une acromicrie par brachyphalangie (au niveau des pouces) ou brachymétatarsie ( $4^{\mathrm{e}}$ ou $5^{\mathrm{e}}$ rayons) donnant des mains et des pieds courts et trapus, associés à des anomalies cervicofaciales (cou court, faciès arrondi lunaire, traits empatés, nez aplati). La PHP s'accompagne d'une hypocalcémie et d'une hypophosphorémie avec des anomalies de la vitamine D, la PTH, le

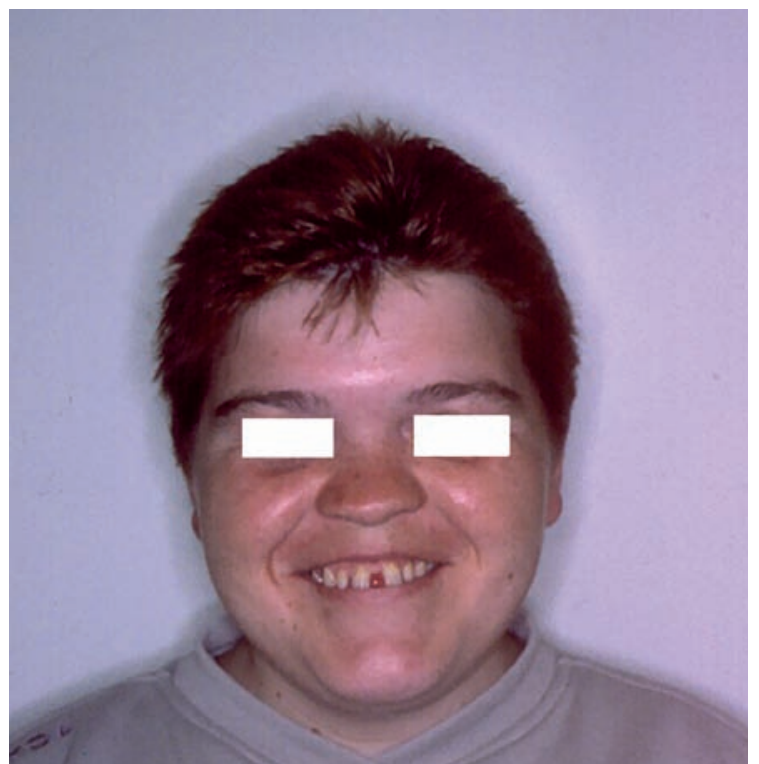

PTHrP (parathyroid hormone-related peptide) et la calcitonine. Des anomalies crâniofaciales et surtout dentaires sont habituellement observées : hypoplasie de l'émail, anomalies du cément, chambres pulpaires larges avec des calcifications intrapulpaires, voire parfois des calcifications intracanalaires $[2,8,11,13,20]$. Une observation typique et particulièrement caractéristique est rapportée ci-dessous.

\section{OBSERVATION}

Elle concerne une patiente âgée de 26 ans présentant une PHP de type la, répondant aux critères cliniques de l'OHA (petite taille, faciès lunaire, cou court, surcharge pondérale...).

\section{Examen clinique}

A l'examen clinique exobuccal, on note un aspect brachycéphale, euryprosope (du grec : eurus, large et prosopis, visage) et transfrontal, avec un étage moyen du massif facial de taille réduite (Fig. 1). A l'examen endobuccal, les arcades dentaires sont larges et les diastèmes généralisés. La patiente présente une macroglossie probablement fonctionnelle. La mandibule est large, arquée, ancrée

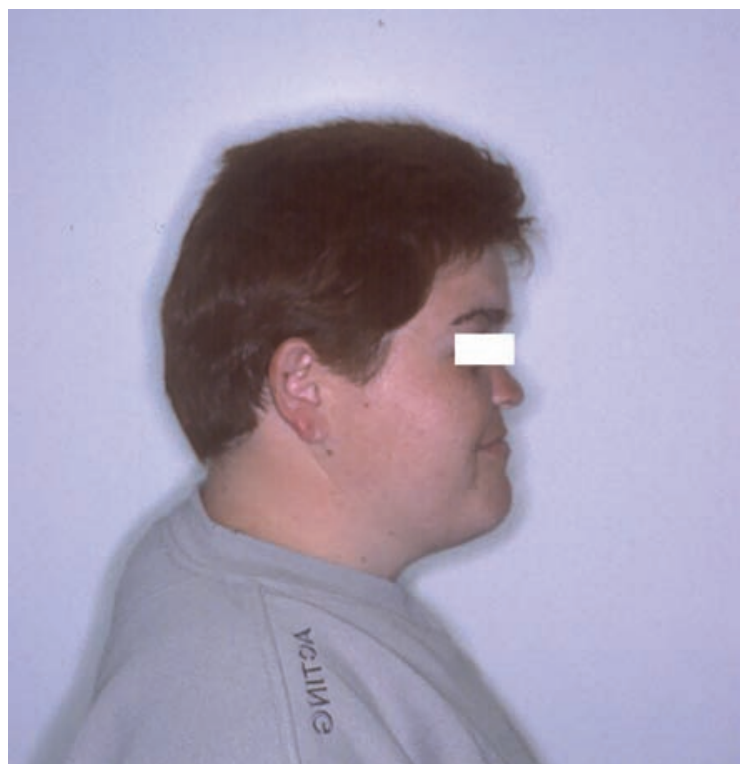

Figure 1 : Aspect clinique de la patiente, de face et de profil : on note le caractère euryprosope, transfrontal et la diminution de hauteur de l'étage moyen du massif facial.

Clinical aspect of the patient in fullface and profile view: we can notice a euryprosope, transfrontal patient with a reduction of the size of the middle part of the face. 
postérieurement. Une absence de calage postérieur bilatéral provoque une propulsion spontanée de la mandibule sans retentissement sur les articulations temporo-mandibulaires. Les dents ont des racines assez courtes avec une chambre et des canaux pulpaires larges. On observe des hypoplasies de l'émail se traduisant par des plages de couleur jaune brunâtre sur plusieurs dents $(11,12,13$, 21, 22, 23) et des caries (17, 24 et 46) (Fig. 2).

\section{Examen radiologique}

Une radiographie panoramique dentaire (Fig. 3) ainsi qu'une radiographie rétroalvéolaire centrée sur 11 et 21 (Fig. 4) ont été réalisées. La 18 est incluse et il n'y a pas d'image de pulpolithe. Une image radioclaire large et peu dense est visible entre les incisives centrales supérieures.

L'analyse des téléradiographies sagittale, axiale et frontale montre une classe III squelettique avec prognathisme ainsi qu'une brachymaxillie et un champ

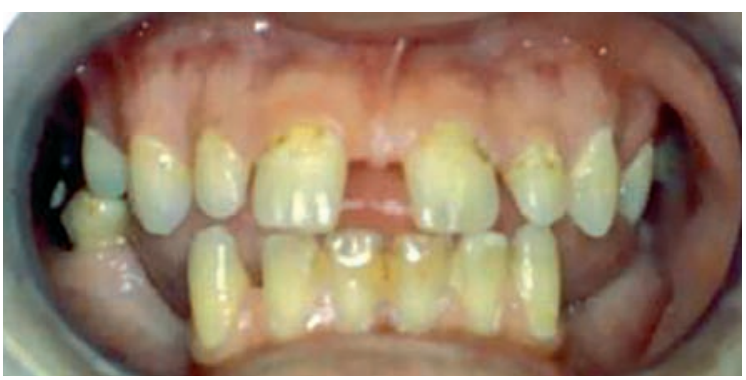

Figure 2 : Aspect de la denture avec occlusion antérieure : présence de diastèmes et d'hypoplasies de l'émail.

Dental aspect with anterior occlusion: presence of diastemas and hypoplasias of the enamel.

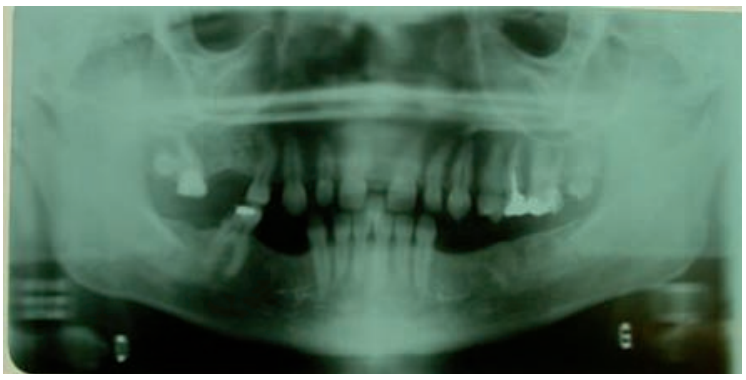

Figure 3 : Radiographie panoramique : édentation postérieure importante bilatérale, dents avec des racines courtes, et une chambre pulpaire large.

Panoramic X-ray : important bilateral posterior edentation, teeth with short roots and broad pulp.

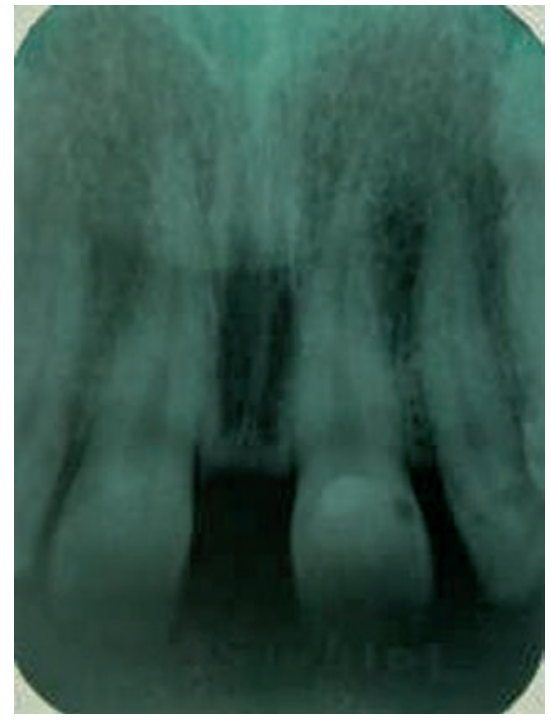

Figure 4 : Radiographie rétroalvéolaire centrée sur 11 et 21 : image radio-claire entre les racines des incisives centrales faisant évoquer un kyste de la papille palatine.

Retroalveolar radiograph between 11 and 21: clear image between the roots of the central incisors corresponding to a probable cyst of the palatin papilla.

mandibulaire développé (Fig. 5) selon l'analyse de Delaire ${ }^{[4]}$. De plus, la patiente présente une pneumatisation importante des sinus maxillaires et du sinus frontal. Les corticales de la voûte du crâne sont denses et épaisses.

\section{COMMENTAIRES}

Ce cas illustre bien les différentes anomalies crâniofaciales et buccodentaires de la PHP de type la. Les anomalies observées seraient la conséquence de l'hypocalcémie, de l'hyperphosphatémie et de la faible concentration plasmatique du calcitriol [22]. Ces anomalies sont également présentes dans l'hypoparathyroïdie où l'on peut observer des anomalies dentaires semblables [11]. Néanmoins, dans la PHP, ces anomalies sont présentes en dépit d'une concentration élevée de PTH immunoréactive circulante, démontrant que la physiopathologie de l'hypocalcémie dans la PHP est différente de celle de l'hypoparathyroïdie.

\begin{tabular}{l}
$\begin{array}{l}\text { médecine } \\
\text { buccale } \\
\text { chirurgie } \\
\text { buccale }\end{array}$ \\
\hline VOL. 12, N ${ }^{\circ} 2$ \\
2006 \\
\hline page 85
\end{tabular}

page 85 

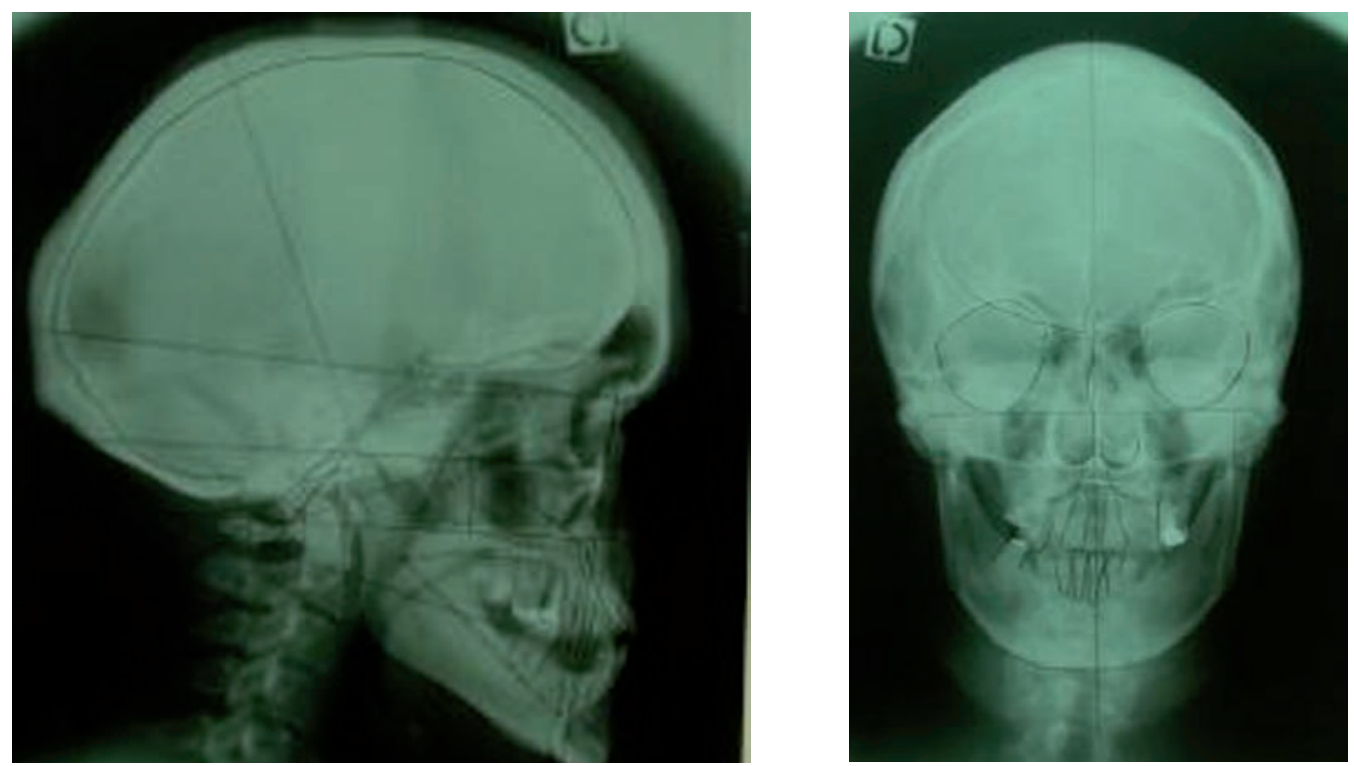

Figure 5 : Téléradiographies sagittale et frontale : classe III squelettique et pneumatisation importante des sinus Saggital and frontal radiograph: skelettical classe III and important pneumatisation of the sinus.

médecine

buccale

chirurgie

buccale

VOL. $12, \mathrm{~N}^{\circ} 2$ 2006

page 86

\section{Anomalies dentaires}

Les dents de la patiente ont une grande chambre pulpaire et des racines relativement courtes. $\mathrm{Ce}$ caractère s'observe communément dans la PHP : chambres pulpaires larges avec des calcifications intrapulpaires, voire intracanalaires, des racines courtes avec un apex émoussé ${ }^{[8]}$.

Les hypoplasies amélaires sont associées à des hypoplasies dentinaires ${ }^{[2,13,20]}$ ce qui se traduit par des taches jaune brunâtre et par une susceptibilité à la carie. Des plages de couleur jaune brunâtre ont été observées chez cette patiente et la susceptibilité à la carie est sans doute responsable en partie de la présence de nombreuses caries, des restaurations dentaires et de son édentation partielle.

La PHP est secondaire à une anomalie du récepteur commun à la PTH/PTHrP, le PTHR1 [26]. Le PTHrP est un peptide de structure analogue à celle de la PTH, qui a été identifié initialement comme facteur responsable d'une hypercalcémie maligne ${ }^{[25]}$. Des études génétiques sur la souris et chez l'homme dans la chondrodysplasie de Bloomstrand, affection où il existe une anomalie du récepteur PTHR1, ont apporté des renseignements sur le rôle du PTHrP; l'inactivation du récepteur PTHR1 démontre qu'un déficit en PTHrP entraîne le plus souvent des troubles dans le développement des seins, de la peau et des dents $[0,20]$.

Chez les fœtus de souris transgéniques pour le récepteur $\mathrm{PTH} / \mathrm{PTH}$ T, on a remarqué que les dents en développement restaient impactées dans l'os alvéolaire, ce qui se traduit par un retard d'éruption [19]. En plus, ces études sur les souris ont mis en évidence le rôle du PTHrP dans la régulation de la formation des ostéoclastes dans le microenvironnement de la dent [17] : il semble contrôler leur turn-over et leur apoptose ${ }^{[3,24]}$. Le PTHrP intervient dans le processus d'éruption de la dent car il est indispensable pour la formation et l'activation des ostéoclastes durant le développement normal des dents $[6,12,14,15,18,2]$.

Chez cette patiente, seule la 18 est incluse mais cette inclusion peut être indépendante de la PHP.

\section{Anomalies crâniofaciales}

Dans I'OHA, on peut observer plusieurs anomalies crâniofaciales. La hauteur de l'étage moyen de la face est diminuée et la mandibule en léger prognathisme. L'ensellure nasale est marquée et les orifices narinaires sont, en général, orientés vers l'avant.

La patiente présente l'aspect caractéristique, observé dans l'OHA, à savoir un aspect brachycéphale, euryprosope et transfrontal ${ }^{[5]}$. Le pro- 
gnathisme résulte de deux composantes: une classe III squelettique et une propulsion spontanée due à l'absence de calage postérieur bilatéral. Les arcades dentaires sont larges avec des diastèmes.

Chez des souris transgéniques, l'inactivation du gène du PTHrP engendre une structure crâniofaciale particulière. L'influence du PTHrP semble être liée à ses effets sur les ostéoclastes mais aussi sur la tension des muscles masseters [21]. La maturation des chondrocytes et la formation de l'os enchondral sont accélérées dans la chondrocrâne (lame criblée de l'ethmoïde, corps du sphénoïde et basi-occipital) et dans les synchondroses ${ }^{7,10,12]}$. De la même façon, dans la PHP, les sutures semblent se fermer précocement, limitant ainsi la croissance suturale. Ces différentes anomalies aboutissent à une platybasie avec épaississement de la voûte du crâne, intéressant surtout la diploë et la table interne ${ }^{[23]}$; ces signes sont retrouvés dans le cas rapporté.

La radiographie rétroalvéolaire faite dans la région des incisives centrales supérieures révèle une zone osseuse peu dense, large, siégeant entre les incisives centrales supérieures, faisant évoquer un kyste de la papille palatine.

\section{RÉFÉRENCES}

1 - Albright F, Burnett Ch, Smith Ph, PARson W. Pseudohypoparathyroidism: an example of SeabrightBantam syndrome. Endocrinology 1942 ; 30 : 922-32.

2 - CROFT LK, WitKop CJ JR, GLAS JE. Pseudohypoparathyroidism. Oral Surg Oral Med Oral Pathol $1965 ; 20: 758-70$.

3 - Chen HL, Demiralp B, Schneider A, Koh AJ, Wang CY, MC CAULEY LK. Parathyroid hormone and parathyroid hormone-related protein exert both pro- and anti-apoptotic effect in mesenchymal cells. J Biol Chem 2002 ; $277: 19374-81$.

4 - Delaire J, Schendel S, TULASNE JF. An architectural and structural analysis: a new lateral cephalometric analysis. Oral Surg 1981 ; 52, 226-238.

5 - DeshaYes MJ. Croissance cranio-faciale et orthodontie (pp. 35-9). Masson, Paris, 1986.

6 - Fukushima H, Jimı E, Kajiya H, Motokawa W, OKabe K. Parathyroid-hormone-related Protein induces expression of receptor activator of NF-(kappa)B ligand in human periodontal ligament cells via a cAMP / protein Kinase A-independent pathway. J Dent Res 2005 ; 84 : 329-34.
L'analyse radiologique montre une mandibule avec un ramus court et large, avec un ancrage condylien situé postérieurement à la norme selon l'analyse de Delaire (milieu M-Oi) (X), avec une brachymaxillie et un champ mandibulaire développé. Ce caractère semble se retrouver chez les souris déficientes en PTHrP : la mandibule est généralement plus petite que chez les souris non traitées, avec un ramus courbé vers le bas [21]. De plus, les sinus maxillaires et le sinus frontal sont très développés, en raison d'une pneumatisation importante.

Chez les patients atteints de PHP, une évaluation systématique de l'état buccodentaire doit être réalisée. Les anomalies trophiques constatées peuvent aussi bénéficier d'une prise en charge générale (apport de calcium et de vitamine $D$ avec une posologie adaptée pour obtenir la normalisation de la calcémie, de la phosphorémie, et de la calciurie, et la réduction du taux de la PTH et du remodelage osseux), mais aussi locale (élimination de tous les foyers infectieux buccodentaires, éventuellement soins dentaires et parodontaux, réhabilitation prothétique adaptée, prophylaxie...).

7 - ISHII-SUZUKI M, Suda N, YAmaZAKI K, KuROda T, Senior PV, BECK F, Hammond VE. Differential responses to parathyroid hormone-related protein (PTHrp) deficiency in the various craniofacial cartilage. Anat Rec 1999 ; 225 : 452-7.

8 - JENSEN S, ILLUM F, DuPONT E. Nature and frequency of dental changes in idiopathic hypoparathyroidism and pseudohypoparathyroidism. Scand J Dent Res 1981; 89: 26-37.

9 - Karaplis AC, He B, Nguyen Mt, Young ID, Seneraro D, OZAWA H, AMIZUKA N. Inactivating mutation in the human parathyroid hormone receptor type gene in Bloomstrand chondrodysplasia. Endocrinology 1998 ; 139 : 52558.

10 - Karaplis aC, luz A, Glowacki J, Bronson RT, Tybulewicz VL JR, Kronenberg HM, MuLligan RC. Lethal skeletal dysplasia from targeted disruption of the parathyroid hormone-related peptide gene. Genes Dev $1994 ; 8$ : 277-9.

11 - KinIRONS MJ, GLASGOW JFT. The chronology of dental defects related to medical findings in hypoparathyroidism. J Dent 1985; 13: 346-9. médecine

buccale chirurgie buccale

VOL. $12, \mathrm{~N}^{\circ} 2$ 2006

page 87 
12 - Kitahara Y, Suda N, Kuroda T, Beck VE, Hammond E, TAKANO Y. Disturbed tooth development in parathyroid hormone-related protein (PTHrp)-gen knockout mice. Bone 2002 ; $30: 48-56$.

13 - Lagarde A, Kerebel LM, KeReBel B. Structural and ultrastructural study of the teeth in a suspected case of pseudoparathyroidis. Biol Buc 1989 ; 17: 109-14.

14 - LIU JG, TABATA MJ, FUJII T, OOMORI T, ABE M, OHSAKI Y, Kato J, WaKISAKa S, IWAMOto M, KuRISU K. Parathyroid hormone-related peptide is involved in protection against invasion of tooth germs by bone via promoting the differentiation of osteoclasts during tooth development. Mech Dev 2000 ; 95 : 189-200.

15 - LIU JG, TABATA MJ, Yamashita K, Matsumata T, IWAMOtO M, KURISU K. Developmental role of PTHrp in murine molars. Eur J Oral Sci 1998 ; 106 (suppl 1) : 143-6.

16 - MARTIn TJ, GILLESPIE MT. Of mice and men, recapitulation of Bloomstrand's chondrodysplasia. Clin Endoc Metab $2001 ; 86$ : 1487-8.

17 - Nakchbandi IA, Weir EE, Insogna KL, Philbrick WM, BROADUS AE. Parathyroid hormone-related protein induces spontaneous osteoclast formation via a paracrine cascade. Proc Natl Acad Sci 2000 ; 97: 7296 300.

buccale

chirurgie buccale

VOL. $12, \mathrm{~N}^{\circ} 2$ 2006

page 88

18 - Philbrick WM, Dreyer BE, Nakchbandi IA, Karaplis AC. Parathyroid hormone-related protein is required for tooth eruption. Proc Natl Acad Sci 1998 ; 95 : 11846-51.

19 - PhILBRICK WM, WYSOLMERSKI JJ, GRALBRAITH S, HOLT E, Orloff JJ, Yang KH, Vasavada RC, Weir EC, Broadus AE, STEWART AF. Defining the roles of parathyroid hor-
21 - Shibata S, Suda N, Yamazaki K, KuRoda T, Beck F, Senior PV, HAMMOND VE. Mandibular deformities in parathyroid hormone-related protein (PTHrp) deficient mice: possible involvement of masseter muscle. Anat Embryol $2000 ; 202: 85-93$.

22 - SILVE C. Pseudohypoparathyroidism syndroms: the many faces of parathyroid resistance. Eur J Endocrinol $1995 ; 133: 145-6$.

23 - SteInBACH HL, Young DA. The roentgen appearance of pseudohypoparathyroidism $(\mathrm{PH})$ and pseudopseudohypoparathyroidism (PPH). Differentiation from other syndromes associated with short metacarpals, metatarsals, and phalanges. Am J Roentgenol Radium Ther Nucl Med 1966 ; $97:$ 49-66.

24 - Suda N, Kitahara Y, Hammond VE, Ohyama K. Development of a novel mouse osteoclast culture system including cells of mandibular body and erupting teeth. Bone 2003 ; 33 : 38-45.

25 - Suva L, Winslow GA, Wettenhall RE, Hammonds RG, Moseley JM, Diefenbach-Jagger H, Rodda CP, Kemp BE, Rodriguez H, Chen EY, Hudson PJ, Martin TJ, WoOD WIA. Parathyroid hormone-related protein implicated in malignant hypercalcemia: clooning and expression. Science 1987 ; 237 : 893-6. mone related protein in normal physiology. Physiol Rev 1996 ; 76: 127-73.

20 - RITCHIE GM. Dental manifestations of pseudohypoparathyroidism. Arch Dis Child 1965 ; 40 : 565-72.
26 - VLAEMINCK-GUILLEM V, WÉMEAU JL. Les pseudohypoparathyroïdies et le concept de résistance hormonale. Presse Med 1999 ; 28 : 1433-47.

27 - WYSOLMERSKI JJ, CORMIER S, PHILBRICK WM, DANN P, Zhang JP, Roume J, Delezoide AL, Silve C. Absence of functional type I parathyroid hormone (PTH) /PTH-related protein receptors in humans is associated with abnormal breast development and tooth impaction. J Clin Endocrinol Metab 2001 ; 86 : 1788-94. 